\title{
Transforming Medical Care: Case Study of an Exemplary, Small Medical Group
}

\author{
Leif I. Solberg, $M D^{1}$ \\ Mary C. Hroscikoski, $M D^{1}$ \\ JoAnn M. Sperl-Hillen, MD \\ Peter G. Harper, $M D^{1}$ \\ Benjamin F. Crabtree, $P b D^{2}$ \\ 'HealthPartners Research Foundation, \\ Minneapolis, Minn \\ ${ }^{2}$ Department of Family Medicine, Robert \\ Wood Johnson Medical School, University \\ of Medicine and Dentistry of New Jersey, \\ New Brunswick, NJ
}

\begin{abstract}
PURPOSE Most published descriptions of organizations providing or improving quality of care concern large medical groups or systems; however, $90 \%$ of the medical care in the United States is provided by groups of no more than 20 physicians. We studied one such group to determine the organizational and cultural attributes that seem related to its achievements in care quality.

METHODS A 15-family physician medical group was identified from comparative public performance scores of 27 medical groups providing most of the primary care in our metropolitan area. Semistructured interviews were conducted with diverse personnel in this group, operations were observed, and written documents were reviewed. Four primary care physician researchers and a consultant then reviewed transcriptions, field notes, and materials during semistructured sessions to identify the main attributes of this group and their probable origins.
\end{abstract}

RESULTS This medical group ranked first in a composite measure of preventive services and fourth and sixth, respectively, in composite scores for coronary artery disease and diabetes care. Our analysis identified 12 attributes of this group that seemed to be associated with its good care quality, with patient-centeredness being the foundational attribute for most of the others. Historical factors important to most of these attributes included small size, physician ownership, and a high value on practice consistency among the clinicians in the group.

CONCLUSIONS The identified 12 attributes of this medical group seem to be associated with its superior care quality, and most of them might be replicable by other small groups if they choose to work toward that end.

Ann Fam Med 2006;4:109-116. DOI: 10.1370/afm.424.

\section{INTRODUCTION}

Tn 2001, the Institute of Medicine (IOM) published a landmark report that decried the large gap between current care quality and what should be achievable, especially for patients with chronic medical conditions. ${ }^{1}$ Other recent studies have confirmed this gap for both the population of the United States as a whole and for the elderly. 2,3 The IOM report called for a transformation in care delivery and identified 6 component aims for quality (safety, timeliness, effectiveness, efficiency, equity, and patient-centeredness). It also identified 6 challenges that care delivery organizations will need to address in that transformation. In 2004, the Future of Family Medicine (FFM) report from all 7 national family medicine organizations also called for a transformation in the way family physicians practice, identifying the need to create and sustain a New Model of practice. ${ }^{4}$ The 11 characteristics of this New Model and the 6 design challenges of the IOM report seem to describe the medical practice needed for the future, at least for family medicine practices. They are listed in Table 1.

Many articles and studies have been published about the ways in which large care-delivery organizations or systems are responding to these challenges. ${ }^{5-10}$ A growing list of publications also addresses the importance of 


\begin{tabular}{l} 
Table 1. The Recommendations for New Care \\
From the Institute of Medicine and the Future \\
of Family Medicine \\
\hline Institute of Medicine \\
1. Redesign of care processes based on best practices \\
2. Use of information technologies to improve access to clinical \\
information and support clinical decision making \\
3. Knowledge and skills management \\
4. Development of effective teams \\
5. Coordination of care across patient conditions, services, and \\
settings over time \\
6. Incorporation of performance and outcome measurements for \\
improvement and accountability \\
Future of Family Medicine \\
1. Personal medical home \\
2. Patient-centered care \\
3. Team approach \\
4. Elimination of barriers to access \\
5. Advanced information systems \\
6. Redesigned offices \\
7. Whole-person orientation \\
8. Care provided within a community context \\
9. Emphasis on quality and safety \\
10. Enhanced practice finance \\
11. Commitment to provide family medicine's basket of services
\end{tabular}

organizational factors and culture for high-quality care, and these publications also focus predominantly on large delivery systems. ${ }^{11-17}$ Yet though $82 \%$ of physicians work in groups of 9 or less (90\% in groups of 20 or less) and the proportion in large medical groups has not changed since $1995,{ }^{18}$ there are only a few studies of the function or culture of small medical groups. ${ }^{18-23}$ These qualitative studies have contributed greatly to our understanding of small practices, especially in terms of complex adaptive systems theory, but we still lack detailed descriptions of any particular smaller medical groups that could serve as exemplary models of transformed care. Without such models, the conceptual exhortations of the IOM and FFM reports will be difficult to translate into real life.

With this need in mind, we noted that several relatively small medical groups in Minnesota were receiving the highest scores on a public performance report card for high-quality preventive and chronic disease care. One group, with the pseudonym Patient First Medical Group (PFMG), agreed to allow us to conduct an intensive qualitative study of its organization and culture. In 2004, this group had achieved the highest score on a composite measure of 10 clinical preventive services for adults among the 27 medical groups providing primary care to most of the residents of the Minneapolis-St Paul metropolitan area. That year, 94\% of all their adult patients were up-to-date for each of the services indicated for them. The group attained the fourth highest score for managing a composite set of 6 coronary artery disease risk factors and the sixth highest score for a composite set of 7 diabetes care and outcome measures. PFMG also has one of the highest scores in the region on patient satisfaction surveys, with $64 \%$ of respondents reporting that they are very satisfied with access to care.

Our goal in this qualitative case study was to provide an analysis and description of the attributes of this exemplary medical group so that other practices and groups interested in achieving similarly high levels of quality might be able to learn from them.

\section{METHODS}

We conducted semistructured interviews in 2 rounds with 16 individuals from each of the 3 PFMG clinics, including the present and former medical directors, managers, supervisors, physicians, nurses, and medical assistants. Selection was primarily driven by the individuals' positions in the organization so we could gather information from each site and each layer in the leadership structure. The original round of 12 interviews elicited information about how the group was organized and how it delivered care, how change was managed, and what the facilitators and barriers to change were. The single interviewer $(\mathrm{MCH})$ also observed clinic operations and reviewed and collected examples of documents related to the study goals. In the second round of interviews, more information was sought from some of the 12 original interviewees plus 4 new ones about how the medical group developed over time, what the financial and business considerations were in its strategies and operations, and how much variation there was among sites and among physicians.

All 11 hours of interviews were tape-recorded and fully transcribed, and the interviewer wrote field notes of her observations and perceptions. These materials and the collected documents were read, reviewed, and discussed in 3 semistructured discussions by the 4 primary care physician coauthors, with consultation support and guidance (by BFC). After identifying observations and themes, the investigators developed them, using an iterative consensus process, into attributes that seemed to be associated with the provision of high-quality care and that we considered likely to be underlying sources of that care. Finally, each feature and characteristic of the 2 models was considered for its relationship to this medical group. Each of these summaries and this article were reviewed with the medical group's leaders for accuracy, but the authors are responsible for any factual or interpretive errors. The study was approved by the HealthPartners Institutional Review Board. 


\section{RESULTS}

\section{Description}

At the time of the interviews, PFMG included 15 family physicians and 3 nurse-practitioners practicing in 3 sites in suburban Minneapolis-St Paul. One nurse-practitioner and 4 to 6 physicians were at each site, which differed primarily in their setting and population. One was in an old suburb with $10 \%$ to $15 \%$ recent immigrants and a more-elderly population, 1 was in an upper middle-class white suburb that was starting to age, and 1 was in a rural setting that was moving toward suburban status. Although each site had some variation, there were far more similarities than differences among them and considerable intermixing of staff and physicians on various committees.

PFMG was established 25 years earlier by 2 new graduates of a nearby family medicine residency. Until 4 years ago, when the physicians decided to sell their practice to a large health plan with a staff-model medical group, the physicians had owned the practice themselves. PFMG chose the sale because they could see no other way to obtain the financial capital necessary for updating their facilities and acquiring an electronic medical record system. As practice owners, they had always paid themselves equally (prorated by full-time equivalents), but at acquisition they converted to a completely productivity-based payment system.

Both before and after the sale, PFMG was managed by a medical director and president, an executive board, and managers for clinical services and patient services, and all major decisions were discussed and ratified by all the physicians in their monthly meeting. After the sale, the medical director reported to the medical director of the staff-model medical group, although until very recently PFMG's operations have continued to be relatively independent of the larger organization.

Clinical staffing patterns were built around clinician-medical assistant dyads, with a front office person connected to each dyad; 1 nurse at each site provided telephone triage and selected patient education or nursing services for the site. All staff members were nonunion. Only about $15 \%$ to $20 \%$ of PFMG's patients were insured by the owning health plan, with the rest of the patients either insured by all other payers in the area, or they were uninsured. The clinical information system has remained entirely paper-based, although in the near future there are plans to implement the larger medical group's electronic medical record.

\section{Attributes}

The main attributes of PFMG that seem associated with its approach to patient care are listed in Table 2. Further description and illustrative quotations from the transcriptions follow. (Quotes are not necessarily given verbatim; some have had minor edits made for readability.)
Table 2. Principal Attributes of Patients' First Medical Group

1. Strong visionary leadership

2. Patient centered

3. Strong support for physician-patient relationship

4. Strong group, team, and standardization orientation

5. Extensive involvement and engagement of all physicians and staff

6. Highly organized change management

7. Focused

8. Strong change and improvement orientation

9. Broad physician sense of ownership and responsibility

10. Market driven

11. Data-based, transparent, and accountable

12. Pride and joy

\section{Leadership}

This physician-owned and -led practice has evolved its medical leadership through an informal consensus process by the entire physician group, but the 2 principal managers share operational leadership. Leadership is strong and consistent at all levels, an approach that could be characterized as both top-down and bottom-up, with an emphasis on leading through example and encouragement. PFMG's leadership approach and remarkably clear vision of its future evolved with time, assisted (according to leaders at each level) by encouragement for leaders to be involved in outside experiences. They note that early on leadership was a matter of individual personality and style, but in the past few years this leadership style has been superseded by a well-developed and increasingly supportive management structure.

"Managers are the agents of change." - Manager

"The way they go about making or deciding about a change is by getting feedback from all areas. It's very seldom just a top-down thing. It's very seldom just a tell that, 'This is the way it's going to be.' Usually we've agreed upon it, and then if you don't do it, now it's a tell." - Manager

"We have a great management team." - Physician

\section{Patient-Centeredness}

The philosophy of patient-centeredness goes well beyond the individual interactions with patients to the operational systems of the clinics. PFMG's story tells of physician unhappiness when they realized that patients seeing more than 1 physician in the group were receiving conflicting advice and messages. To reduce this source of patient confusion, they deliberately developed an increasingly standardized approach to care. Another aspect is clinician-patient continuity, which PFMG actively encourages but leaves up to the patient with no disagreement among clinicians, as there is a tradition of distributing work opportunities equally. 
Patient-centeredness was cited by many personnel as the purpose of their work, with the goal to improve the care that patients receive.

"Every day that we walk in this door, we say to ourselves we're going to give the best that we can of ourselves in making the patient satisfied, from the time we answer the phone or the time they walk in the door to the time we finish the task that they've asked us to do." - Manager

"Without being able to deliver a good-quality patient care, what would be the sense of changing everything?" - Manager

"We're forever attached to our patients [following up after a visit]." - Medical Assistant

"They [patients] know we know who they are." - Manager

"We want our patients, from the moment that they walk in the door, to feel everything is very seamless." - Manager

Focus on the Physician-Patient Relationship Most of the interviews included examples of the strong focus on making the interaction between the patient and physician as efficient and effective as possible. Although PFMG has a paper charting system, it is extremely well organized and up-to-date, allowing for efficient use. Each site has a "chart-prepper" whose sole duty is to review and organize the chart for each scheduled patient so that all results are entered, problem lists and data flow charts are filled in, preventive or chronic disease services needed are highlighted, and patient education materials that might be needed are included. The chart is further reviewed and extended by the medical assistant, who circles abnormal vital sign values in red and completes test orders or referral forms to make it easy for the physician to sign them. The after-visit summary results provide both patients and physicians the same benefits after the visit. It was interesting to find relatively little use of standing orders or clinical task delegation in this group, which relied instead on extensive involvement of all other staff to bring together information that would prompt the physician's actions. Apparently this tradition stems in part from not having nurses in the practice until recently, although now their protocol-driven care roles (eg, in contraceptive management) are steadily expanding.

"If my MA [medical assistant] sees an eye exam is overdue, she'll already have a referral on the front of the chart for me." - Physician

"That's kind of our way of making sure everything is in the chart ready for the doctor." - Medical Assistant

"If Dr. X has numbers that are low and that's the doctor who I'm supposed to support, what can I do to help make that work better?" - Physician
Broad Physician Sense of Ownership and Responsibility All the physicians in the group seem to feel responsible for both clinical and administrative aspects of the practice. No quality improvement or guideline activity is undertaken without a physician champion who is responsible for teaching colleagues about it and arguing for the care approach being suggested. Similarly, all are involved in supporting the arrangements made to ensure that the approach decided upon is implemented well.

"We feel as if our presence here as providers [physicians] allows these people to be employed. And so there's this sense of responsibility about that." - Physician

"Each committee has a provider and it's up to that provider to bring it to the provider group to discuss it amongst all the providers and then decide." - Supervisor

"When [physicians] decide to make a change, they look at the global reasons for making the change." - Manager

"If you don't have the provider support [for a change effort], it's just going to crumble. We have really good provider support here." - Supervisor

Extensive and Intensive Involvement and Engagement The interviews show strikingly consistent comments and descriptions, from director to medical assistant, of the mission, vision, values, and strategies, as well as of how change is managed. They all appear to feel involved in these actions as the result of an explicit practice of not making any change without involving those whose work it affects.

"I just happened to be on the [design] team, but it could have been any patient service employee here ... it would have been her responsibility to get that info to me and the other sites as well." - Supervisor

"One of the patient services supervisors is the visit summary guru." - Manager

"You know you can go to [managers] if you have questions or problems or ideas? Oh yeah. Definitely." - Medical Assistant

\section{Highly Organized Change Management} When first looking at the PFMG organizational chart, we were impressed by its apparent complexity and the extensive interlocking and overlapping committee structure. There is a practice leadership team that involves both medical and managerial leaders, as well as a monthly physician meeting, where the real policy decisions and changes in care are addressed. A Continuous Quality Improvement committee meets monthly and oversees separate committees for laboratory, forms, quality improvement change agent, safety, and quality control. The large multidisciplinary and multisite design 
team is responsible for planning implementation of major policy initiatives, such as an after-visit summary used to monitor and address all follow-up services and arrangements. In addition, previous major initiatives, such as the group's conversion to advanced access appointment scheduling 3 years earlier, still get maintenance attention from an access team that meets quarterly. All this activity is in addition to regular meetings of managers, medical assistant staff, patient services staff, nurses, etc.

Despite this organizational complexity, everyone we interviewed appeared to understand the whole system, knew how to interact with it in a meaningful way, and was confident that good decisions would be made. It also seemed that everyone in this practice is a systems thinker to an unusual degree. In developing and implementing change, extensive use is made of true pilot tests, measurement, feedback, revision and refinement, diverse communications, and attention to maintenance. Finally, leaders of committees or teams strive to make meetings productive and engaging, working to hone needed skills.

"We RNs [nurses] pilot it, see how it goes, survey patients, make a few changes, then start it at another site, and then the third." - Nurse

"It's supposed to start with the committee, go to the providers, come back to the committee, rework it." - Supervisor

"That's how pretty much we start everything, is flow-charting it and looking at it and seeing exactly, 'Is this step necessary? Why do we do this?' - Supervisor

\section{Focus}

Unlike many medical organizations, PFMG avoids the trap of trying to improve too many things at one time with the result that none is done well. Decisions on new initiatives are made selectively and sparsely by the leaders and physicians, concentrating on those changes that will make the most difference to patients. In the clinical area, they worked only on diabetes for 3 years until they got it right; now they are addressing coronary heart disease and depression. The improvement strategy for a given clinical topic is distilled to a simple operational strategy that is easy to communicate. For example, the initial major operational strategy to improve preventive services and diabetes was to develop a previsit planning process. The current major initiative to improve care is implementation of a carefully designed and pilot-tested after-visit summary. As is their chart system, the after-visit summary is paperbased, and copies are produced for the chart, patient, and the designated patient services employee. The patient services employee is responsible for tracking everything ordered through completion and noting it in the chart; an added benefit is making patient services employee feel importantly involved in patient care.
"We want to capture the patients that really need to have these [after-visit summary] instructions." - Manager

"We haven't been doing [after-visit summaries] for quite a year.... I could see us making yet another change to this. So we're constantly following up, you know?" - Manager

"Somebody in the organization has to have a really good understanding of how long it takes to make a change, how long it takes to communicate, and the learning curve of the change. If you have too many really big changes, you totally overload the system and the people that need to implement it, and they're going to get frustrated. And once you've started implementing a change, leadership needs to stay engaged in the process, constantly be following up." - Manager

\section{Strong Change and Improvement Orientation}

At all levels, those in PFMG appeared to feel invigorated by change rather than threatened by it. In part, it is clear to everyone that change is needed and is driven by both a business and quality mission. It also appears linked to feeling that the changes are under the control of the whole group as well as each employee.

"When we start to do something new, people really cling to that change and want to do it and see how it works. They're open to change." - Medical Assistant

"We really want change, and our provider on this particular committee is very aggressive when it gets to change..., but it was the whole team pushing him, too, to get this done and get it to where we want it to be."

- Supervisor

"Over time [change] developed as just a core value of quality within [PFMG]." - Manager

"You're going to have to change so much, but in the long run it's going to end up being easier for you, so you just have to get adjusted to it." - Supervisor

\section{Teamwork and Standardization}

Standardization often has a negative connotation among physicians, but these physicians and staff members seem to accept that locally tailored standardization and teamwork are the keys to improvement and to providing a consistently high quality experience for each patient. Teamwork was an early goal in this group's history, and they have used many techniques to foster it, including equal pay for the physicians, a common office area at each site for all clinicians, carefully selecting new clinicians who fit the team model, providing new personnel with mentors and extended orientation, publicizing internally both site and clinician performance measures, using extensive involvement and communication, and sharing stories that demonstrate these goals. In fact, the group has consciously used its approach to hiring and retention of clinicians and other staff to foster teamwork and standardization, as well as the other attributes. 
"We're all in this together, and every one of our roles is just as important ... it would be like functioning without one of your limbs." - Manager

"The culture of the providers is that they all will do what's been decided will be done ... aren't any loose cannons." - Manager

"Everyone at this clinic seems to be pretty committed to following the guidelines." - Physician

"Not letting individual providers make specific little tweaky rules [about access]." - Manager

"It's a team effort, truly, when we make a change. It's all departments and all areas being aware of the change and why we've decided to make it that make that change a successful one." - Supervisor

\section{Market Driven}

More than 13 years ago, as the local marketplace was becoming dominated by a few health maintenance organizations and large medical groups, PFMG decided that if they were to survive and thrive as an independent group, they needed a desired distinguishing feature. That feature was to be quality. Not that they would necessarily be the first to come up with new approaches to care, they would be the best at implementing them. Thus they value agility and change highly. They also have been solidly committed to the local quality improvement collaborative (Institute for Clinical Systems Improvement [ICSI]) from its inception, which they consider a valuable source of assistance in their quest for quality.

"I think from a patient perspective quality is the whole experience.... If we don't have people that want to continue coming here and find it pleasurable and delightful, basically we don't have a business." - Manager

"What we recognized at that point was that, as a small group, we had certain areas where we could have the competitive advantage that we needed to remain a viable business. Part of that was cost, and more and more a big part of it was quality." - Physician

\section{Data-Based, Transparent, and Accountable}

It appears that every change initiative, clinical or administrative, is based on repeated measures of its implementation or outcome. The results of these measures are posted and used to compare physicians as well as sites, with the goal of learning from variation and not squelching it unnecessarily. It is also clear there are no secrets and everyone must be accountable for patient care. For example, each month, the medical assistant supervisor audits the charts of each medical assistant to maintain their systems and to identify areas needing attention.

"By doing the measurements and publishing the measurement, there's a certain amount of pressure." - Manager
"I'm pulling ... probably 120 charts a month [to audit MA work]." - Supervisor

"Some minor variation [is] appropriate as long as there's enough transparency about it so ... they are at some level accountable for the impact of the variation." - Physician

"We do have everyone read it [memos or committee minutes], sign off that they've read the information." - Manager

"There's just people that get into habits or don't really like change, and we're working on them.... On the back of each door in every patient room, it says, 'Did you get a visit summary from your provider today?' Now the patients are asking the providers for one." - Supervisor

Pride and Joy

Although hard to quantify, this attribute comes through in every interview. All physicians and staff appear to be proud of what they are accomplishing and take joy in their continuing work lives.

"That's what everybody likes about this clinic and working here, too, is the fact that we have so many responsibilities and so many [different] duties." - Supervisor

"The relationship between the providers and the staff has always been very open and strong. There's a lot of mutual respect and working together." - Manager

\section{History or How Did They Get This Way?}

When interviewed, 1 of the 2 practice founders emphasized that from the beginning they were determined to combine high-quality medical care with being family friendly for physicians and staff, and they wanted an egalitarian structure to the work environment. Those emphases appear to have continued, shaping their early realization that patients found confusing the varying messages when they saw different clinicians. Through extensive discussions in their monthly all-clinician meetings, they made a conscious decision that the only solution was to standardize their care as much as they could while maintaining individual tailoring in accordance with patient desires and needs. They also found that consistent, standardized care was impossible to achieve in the absence of practice systems to support it. About 10 years ago, they decided to take extensive advantage of the quality improvement knowledge and skills available through a regional quality improvement organization (ICSI). Participation in ICSI allowed them to interact with leaders from other, more-sophisticated, large medical groups, which led to an expanded world view of the importance of quality as a key business advantage in a competitive market. Finally, the highly structured and broadly involved management was strengthened by hiring a new administrative leader with such interests. 


\section{DISCUSSION}

PFMG appears to be a remarkable medical practice. It is especially interesting that it has reached this point with limited resources and without the electronic information systems that so many consider a prerequisite for good care. Although we have only the public performance measurements to compare this group quantitatively with other medical groups in the metropolitan area, the composite nature of these measurements means that the 3 measures described actually reflect 23 different quality measures. PFMG's ranking regionally is all the more remarkable considering that the level of quality in most groups in this area is well above national averages. Health Plan Employer Data and Information Set (HEDIS) rankings for the PFMG effectiveness-of-care measures (representing an average across all medical groups providing care to its members) make this health plan one of the top 5 for quality in this region of the country. ${ }^{24}$

How well does this group seem to fulfill the IOM challenges and the FFM characteristics? Although they have not achieved perfection, primarily in the areas of information technology and finances, they are at least satisfactory models for other medical groups in all of the other items. It is also hard to evaluate their current lack of electronic information technology, because their many creative paper tracking systems and excellent paper chart seem to accomplish well many of the same goals. They are scheduled to implement an electronic medical record in 2006 that will include a variety of patient Web-accessible features and a secure electronic communication system with patients. Finally, most of the attributes we identified as central to the culture and success of PFMG are not mentioned by the IOM and FFM, perhaps because they wanted to emphasize patient-perceived characteristics rather than the background group function that allowed those behaviors to happen. We believe that these unrecognized attributes of its organizational culture are what has allowed PFMG to create a practice that meets so many of the design recommendations of national bodies and might even be required as a foundation for achieving those recommendations. Certainly, most experts who have studied successful organizations have concluded that culture is absolutely critical to successful change. ${ }^{25-29}$ The point is most explicitly made by Senge et al: "The fundamental flaw in most innovators' strategies is that they focus on their innovation, on what they are trying to do rather than on understanding how the larger culture, structures, and norms will react to their efforts." ${ }^{10}$ The lessons from PFMG should at least be considered by those who wish to transform their care for the future.

What accounts for such an exemplary organization? Clearly, having homogeneous single-specialty profes- sionals who are financially and medically responsible for the practice helps, as does its origin as a new practice with a mission focused on providing high-quality care. These characteristics, however, are true of many primary care practices in the United States.

After considerable discussion, we think that there are 2 root causes for this practice taking a path different from most-leadership and patient-centeredness. In this case, patient-centeredness goes well beyond that of individual clinicians for individual patients; instead, it is a system-level attribute for their entire patient population. This unusually strong focus on patients resulted in the willingness of PFMG to standardize care, implement common guidelines, and acquire many of the other attributes we identified. Those attributes would have been only good intentions had PFMG not had unusually capable leaders who were able to successfully involve and engage the entire staff in implementing them, even though the leaders might not have seen the path clearly from the start. They certainly do now, as reflected in the current medical director's insightful comment: "The secret to meeting [quality] goals is to systematize things so they become automatic, and less thought is needed to make sure each item is completed."

Casalino et $\mathrm{al}^{9}$ studied more than 1,000 medical groups of more than 20 physicians and found that 2 factors most strongly predict what they called "care management processes" for chronic disease care: external incentives and information technology. Bodenheimer et a ${ }^{15}$ subsequently followed up this study by interviewing 158 leaders of 15 diverse medical groups to identify the principal facilitators and barriers to the implementation of such care management processes. They found that the 2 most commonly mentioned facilitators were strong leadership and an organizational culture that placed a high value on quality. While inadequate information technology and reimbursements unrelated to quality were frequently mentioned barriers, the other 3 main barriers cited were a poor financial position, physician resistance, and physicians being too busy.

Our region has had financial incentives for attaining high scores on quality measures since 1997, and interviews with PFMG personnel suggest that these incentives did contribute to the group's focus on quality improvement. Electronic internet technology has not been necessary for this group, however, as they have been able to make good use of excellent paperbased information systems. Their experience suggests that their organizational mission and culture focused strongly on patients and quality overcame physician busyness and resistance, although at least minimal financial security may be needed.

We are aware of the limitations of such a case study. Our information was incomplete and subject to the 
biases of the investigators and reporters. If there are mistakes, however, they are ours, and we report our analysis in the spirit of raising hypotheses for further research rather than as definitive answers. In addition, we have no way of knowing whether these same attributes are found in other medical groups with quite different performance results. Finally, one might question whether this group is truly successful in that it chose to sell itself to a larger organization. It did so because it lacked the capital to invest in increasingly necessary major expenses, such as an electronic medical record and building space and renovations. This outcome may have been more likely in our region, which has limited reimbursement for primary care services, but it is a cautionary tale for even such excellent groups.

Nevertheless, we agree with Fernandopoule et $\mathrm{al}^{12}$ that we need to learn how to identify high-performing organizations, better understand what differentiates the successful from the unsuccessful ones, elucidate the process of organizational change, and explore the role and characteristics of good leadership. As they said, case studies can "provide essential qualitative insight into attributes associated with quality performance." We believe that PFMG illustrates the truth of that statement.

To read or post commentaries in response to this article, see it online at http://www.annfammed.org/cgi/content/full/4/2/109.

Key words: Delivery of health care; efficiency, organizational; group practice; patient satisfaction; physician's practice patterns; preventive health services; quality assurance, health care

Submitted April 28, 2005; submitted, revised, August 2, 2005; accepted August 25, 2005.

Funding support: Supported by Grant \#041868 from The Robert Wood Johnson Foundation

Acknowledgments: We thank all the physicians and staff at North Suburban Family Physicians who cooperated with this effort to describe their approach. We are especially grateful for the contributions of Patrick Courneya, MD, Karen MacKenzie, MD, Marcie Quiring, and Sherry Behm, RN, who facilitated the interviews and reviewed our findings without any effort to influence them.

\section{References}

1. Institute of Medicine. Crossing the Quality Chasm: A New Health System for the 21st Century. Washington DC: National Academy Press; 2001.

2. McGlynn EA, Asch SM, Adams J, et al. The quality of health care delivered to adults in the United States. $N$ Engl J Med. 2003;348:2635-2645.

3. Wenger NS, Solomon DH, Roth $\mathrm{CP}$, et al. The quality of medical care provided to vulnerable community-dwelling older patients. Ann Intern Med. 2003;139:740-747.

4. Martin JC, Avant RF, Bowman MA, et al. The Future of Family Medicine: a collaborative project of the family medicine community. Ann Fam Med. 2004;2(Suppl 1):S3-S32.

5. Perlin JB, Kolodner RM, Roswell RH. The Veterans Health Administration: quality, value, accountability, and information as transforming strategies for patient-centered care. Am J Manag Care. 2004;10:828-836.
6. Miller RH, Bovbjerg RR. Efforts to improve patient safety in large, capitated medical groups: description and conceptual model. J Health Polit Policy Law. 2002;27:401-440.

7. Feachem RG, Sekhri NK, White KL. Getting more for their dollar: a comparison of the NHS with California's Kaiser Permanente. BMJ. 2002; $324: 135-141$

8. Rundall TG, Shortell SM, Wang MC, et al. As good as it gets? Chronic care management in nine leading US physician organisations. BMJ. 2002;325:958-961

9. Casalino L, Gillies RR, Shortell SM, et al. External incentives, information technology, and organized processes to improve health care quality for patients with chronic diseases. JAMA. 2003;289:434-441.

10. Sperl-Hillen J, O'Connor PJ, Carlson RR, et al. Improving diabetes care in a large health care system: an enhanced primary care approach. Jt Comm J Qual Improv. 2000;26:615-622.

11. Bender AD. The organization of the medical practice and implications for delivering quality care. Qual Manag Health Care. 1995;3:47-53.

12. Fernandopulle $R$, Ferris $T$, Epstein $A$, et al. A research agenda for bridging the 'quality chasm.' Health Aff (Millwood). 2003;22:178-190.

13. Landon BE, Reschovsky J, Reed M, Blumenthal D. Personal, organizational, and market level influences on physicians' practice patterns: results of a national survey of primary care physicians. Med Care. 2001;39:889-905.

14. Vaughn TE, McCoy KD, BootsMiller BJ, et al. Organizational predictors of adherence to ambulatory care screening guidelines. Med Care. 2002;40:1172-1185.

15. Bodenheimer T, Wang MC, Rundall TG, et al. What are the facilitators and barriers in physician organizations' use of care management processes? Jt Comm J Qual Saf. 2004;30:505-514.

16. Shortell SM, Zazzali JL, Burns LR, et al. Implementing evidence-based medicine: the role of market pressures, compensation incentives, and culture in physician organizations. Med Care. 2001;39:162-78.

17. Gandjour A, Kleinschmit F, Littmann V, Lauterbach KW. An evidencebased evaluation of quality and efficiency indicators. Qual Manag Health Care. 2002;10:41-52.

18. Casalino LP, Devers KJ, Lake TK, Reed M, Stoddard JJ. Benefits of and barriers to large medical group practice in the United States. Arch Intern Med. 2003;163:1958-1964.

19. Miller WL, McDaniel RR, Jr., Crabtree BF, Stange KC. Practice jazz: understanding variation in family practices using complexity science. J Fam Pract. 2001;50:872-878.

20. Miller WL, Crabtree BF, McDaniel R, Stange KC. Understanding change in primary care practice using complexity theory. J Fam Pract. 1998;46:369-376.

21. Crabtree BF, Miller WL, Aita VA, Flocke SA, Stange KC. Primary care practice organization and preventive services delivery: a qualitative analysis. J Fam Pract. 1998;46:403-409.

22. Crabtree BF, Miller WL, Stange KC. Understanding practice from the ground up. J Fam Pract. 2001;50:881-887.

23. Tallia AF, Stange KC, McDaniel RR, Jr., et al. Understanding organizational designs of primary care practices. J Healthc Manag. 2003;48:45-59; discussion 60-41.

24. The State of Health Care Quality: 2004. Industry Trends and Analysis. Washington, DC: National Committee for Quality Assurance; 2004. Available at: www.ncqa.org/communications/SOMC/SOHC2004.pdf.

25. Collins J. Good to Great. New York, NY: HarperCollins Publishers Inc; 2001.

27. Schein E. The Corporate Culture Survival Guide. San Francisco, Calif: Jossey-Bass; 1999.

28. Senge PM. The Fifth Discipline: The Art \& Practice of the Learning Organization. New York, NY: Currency Doubleday; 1990.

28. Kotter JP. Leading Change. 1st ed. Boston, Mass: Harvard Business School Press; 1996.

29. Silversin J, Kornacki MJ. Leading Physicians Through Change. Tampa, Fla: American College of Physician Executives; 2000.

30. Senge PM, Kleiner A, Roberts C, et al. The Dance of Change. New York, NY: Doubleday; 1999. 